\title{
Technology in Accounting: Social Media as Effective Platform for Financial Disclosures
}

Daniel H. Boylan. Widener University. USA. danboylan2312@ hotmail.com

Cavan L. Boylan. Purdue University. USA

\begin{abstract}
Many people have embraced social media (SM), including both personal and professional uses. Corporations hoping to capitalize on this indoctrination are seeking to understand ways they can engage in $\mathrm{SM}$. One possible idea is disseminating financial results. This topic emerged as a result of the recent decision by the Securities and Exchange Commission granting permission. A problem for company leadership is the lack of understanding of the potential SM has to enhance shareholder value. Little research exists today on the impact of a corporate policy to use social web to announce dividend or earnings statements on shareholder value. This research studied SM platforms for financial communications on stock price. By calculating cumulative abnormal returns, researchers learned the impact of SM for financial disclosures. The sample size included thirty-four publicly traded American financial institutions. This research seeks to advance understanding of financial statement dissemination using SM.
\end{abstract}

Keywords: Disclosure, Social Media, Stock Price

\section{INTRODUCTION}

The use of SM has revolutionized communications in the last decade, including the availability of corporations to directly engage their stakeholders. SM allows 
companies the ability to provide information in a real time format that includes the content they wish to communicate.

Once thought of as a toy for youth, SM has developed into a multi-faceted network of information platforms that connect interested parties. Many individuals have adopted technology in both their personal and professional lives. This provides an opportunity for an organization to engage constituents on a variety of topics including investor relations, marketing, sales, public relations, and financial statement disclosure. Adapting these policies connects the organizations to the wants and use patterns of those it is trying to reach (Deisler \& Newton, 2013).

Historically, organizations were reliant on third parties to communicate their information or mailing the Annual Report, Form 10-K, and Form 10-Q. For many organizations this was not effective due to cost, speed, and the control of content. The cost relates to the mailing for these documents to potentially millions of interested stakeholders. Speed refers to the organizations quickness in delivering information. Control of content relates to an organization's inability to control the message presented to the public. Upon receipt, many large news agencies handpicked what information they communicated. That meant organizations were at risk of having only a portion of their message communicated or not having their message communicated at all. This is especially hard for small local organizations that do not have a national recognition. They often found they weren't significant enough to have their information communicated. This leaves these local companies at a competitive disadvantage in building their stakeholder base.

Just because additional methods of dissemination are available, doesn't mean that they provide a benefit to an organization. This research has an objective of determining if shareholder value is enhanced by such communications. This research has the following research question: do SM based financial disclosures improve shareholder value in financial institutions?

The remainder of this work is organized into the following four sections. First, there is a literature review looking at prior literature that informs this study. The next section includes methodology, including both the research question and experiment design. The third section includes the research findings. Finally, the research ends with conclusions, including limitations and future research. 


\section{LITERATURE REVIEW}

Organizations are both required and desire to communicate certain financial information to interested parties. Most commonly, this process is started with the earnings release. The earnings release involves several entities and individuals. These include a company's internal and external accountants, lawyers, public relations representatives, and other executives.

Upon completion of the earnings release, organizations have a variety of methods for communicating to interested stakeholders. These include the traditional methods of utilizing a news intermediary, the corporate website, and SM to name a few. The selection of how information is communicated is very important to a company. Organizations are constantly looking at matching the methods used to disseminate financial statements with the stakeholders they wish to reach. The figure below represents several possible methods organizations can use to reach different targeted stakeholders. Though numerous methods exist, three methods are displayed. They include the use of news intermediaries, the organization's website, and the organization's SM platforms, as follows:

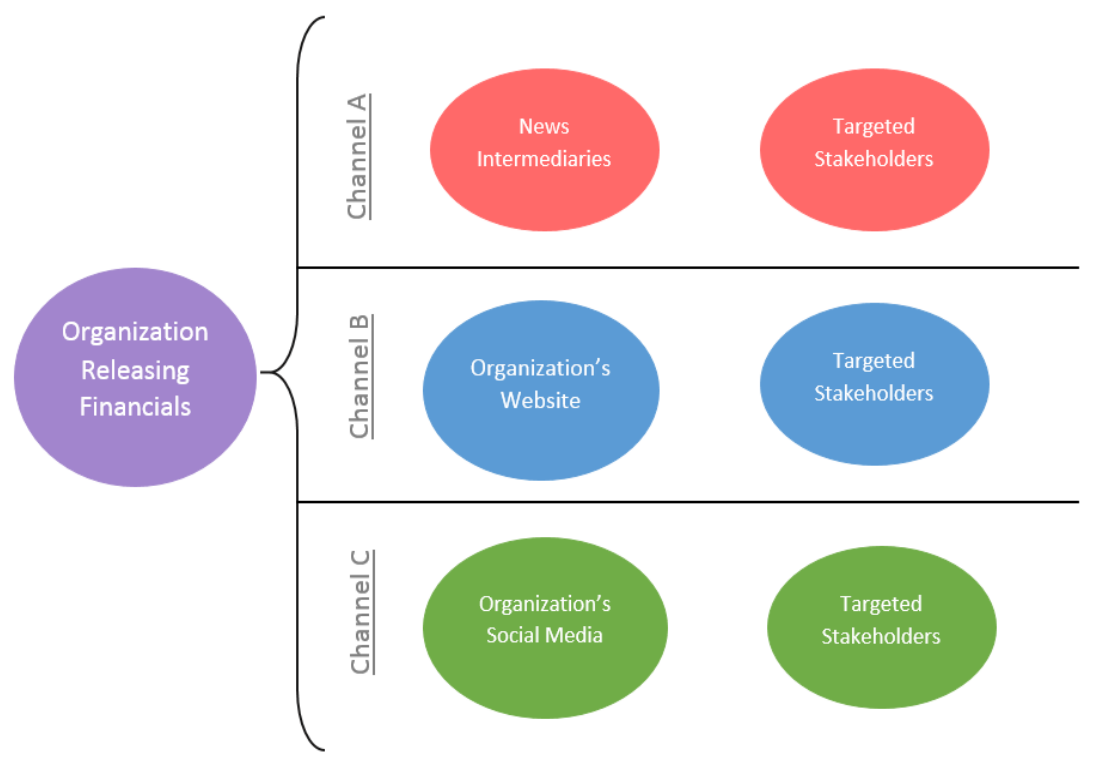

Figure 1. Examples of three possible distribution channels for organizations releasing their financials to different targeted stakeholders

This research is focused on the use of SM. For SM dissemination to be successful, it must entail several objectives. First, the platform must be a legally viable 
option. Second, the medium must be desired by stakeholders. This includes that those interested parties have both adopted the technology and are willing to use it. Third, the company should feel like they are better able to control the information released.

When looking at the legal viability, there has been a trend towards allowing organizations additional flexibility in the dissemination of their financial statements. The Securities and Exchange Commission added flexibility for organizations in 2008 by allowing financial dissemination via corporate websites. This allowed organizations to cheaply communicate and provided the ability to make corrections. In 2013, the SEC permitted the use of SM for such communications (SEC Clarifies, 2013). Social networking offers a chance for organizations to engage with their stakeholders and possibly reach new investors; organizations need to have a deeper understanding of the power of such communication (Morgan, 2013).

SM platforms are an acceptable means to directly interact with those interested in an organization (Alexander \& Gentry, 2014). This acceptance gains importance as traditional media sources are in decline. Organizations that do not adopt a strategy that includes SM risk not reaching the potential investors. The SM presence does not have to be extensive. In fact, those organizations that have focused and less visible SM policies draw the greatest benefit (Ramassa \& Di Fabio, 2016; Blankespoor et al., 2014).

When looking at the desirability by stakeholders, SM is the strongest new platform when considering factors necessary for success including: stakeholder adoption, organization usage, and future impact. Adoption of stakeholders is relatively high considering, roughly $33 \%$ of investors older than 65 years old use SM, while over $70 \%$ of investors under 40 years old prefer a SM presence (Charlier, 2013). Organizations have also engaged SM. The percentage of Fortune 500 companies on various SM is: Twitter 77\%, Facebook 70\%, YouTube $69 \%$, Google+ 35\%, and Instagram 44\% (Barnes et al., 2013). Many companies engage more than one social network to ensure communications are effectively reaching their target audience (Heggestuen \& Danova, 2013; Reilly \& Hynan, 2014). When determining future impact several key facts are important. First, the largest group of adopters is the age group of 35 - 44 year olds. Companies are interested in reaching targeted likely investors (Kaplan \& Haenlein, 2010). 
Second, as younger age groups join investor pools, reliance on social networking and their need for a strong personal connection is an important part of social networking engagement. Companies engaging customers should capitalize on this need by ensuring communications provide a feeling of connection or belonging. Time commitment, reader intensity, such as blogging or "Retweeting," and willingness to engage the organization are several ways a company can measure effectiveness ( $\mathrm{Li}$ et al., 2014). Typical users often willingly engage with organizations they have an interest in, make it important for organizations to be selective about the platforms they use (Li et al., 2014).

When looking at the organization's ability to control the information released, organizational control of information is an added benefit of distribution. Rather than relying on the traditional forms of releasing financial information to a thirdparty news organization, an entity can control the content deciphered by their own reporting using SM. Often third-party news organizations, motivated by profit maximization, do not fully cover some organizations. Other organizations are too small to have a national news agency report their information. SM tactics empower entities in the release of their information. This control of information includes: who receives, how they receive, and how much information they receive (Schumaker \& Chen, 2009).

For many organizations bad news can be devastating. Control of information also entails the ability to manage downside impact of negative news such as bankruptcy or loss of a key executive. Experts call this idea the pessimism effect, which states a small decrease in stock price is statistically significant for organizations. For example, in one study, researchers found that negative words used by a corporation could have a negative impact on share price by several percentage points (Tetlock, 2007).

Organizations should target specific individuals they wish to engage and communicate in the manner those stakeholders expect (Boylan, 2016). For example, many organizations use social networks to communicate marketing or public relations information. During an emergency, social web is a powerful tool to communicate information (Alexander \& Gentry, 2014).

The SM most attractive for financial markets are the same platforms that are most embraced by stakeholders and adopted by organizations. These include Twitter, LinkedIn, and Facebook (Ramassa \& Di Fabio, 2016). The reason for this 
attractiveness is the ability for an organization to create a message in a way that other users will help them spread the message. Companies often design messages that can be co-created. This is achieved when information is designed in a manner to allow further sharing by "Retweets," blogs or posts (Deisler \& Newton, 2013). This added communication magnifies the impact of the original message (Thoring, 2011).

Organization's engage new dissemination techniques for several reasons. Companies often engage technologies their competitors adopt (Charlier, 2013). As one organization adopts a SM platform, competitors will follow. Organizations that have enough information about proper manners of adoption would encourage leaders to engage in nontraditional financial communication (Deisler \& Newton, 2013).

This research studies an emerging trend. Companies can reach a wider base of constituents via the social web. Roughly seventy percent of companies do not engage social networking to scatter their financial results. Many organizations use a SM platform to communicate in various roles including: marketing, public relations, and employment to name a few. It is not clear whether adding financial distribution to the SM use policy would benefit an organization. As a result, companies have a strong interest in research that may encourage them to reevaluate policies for the use of social networking (Charlier, 2013).

\section{METHODOLOGY}

This research focused on both financial disclosure and technology applications. The purpose of this study was to discover the impact of a corporate financial release using the social web and the resulting change in the organization's stock price. A change in law created an opportunity for companies to capitalize on the capacity to use social networks to connect with parties interested in their information. The independent variable is the use of SM. The dependent variable is the change in shareholder value as determined by the rise and fall in stock price.

This research is an event study. It compares stock price changes of financial institutions for multiple time periods. Two sets of independent variables compared the use of SM and the nonuse. A Cumulative Abnormal Return (CARs) method was engaged. This CARs measures stock prices both before and after an earnings announcement, impacting the dependent variables. Researchers used several data 
banks to compile information. These data banks included the Federal Deposit Insurance Company (FDIC), Federal Reserve Bank of Chicago, and the Consumer Federation of America. Though accurate, the data from the FDIC needed to be verified and validated for this research. This included a review of SM feeds, ticker symbols, the beginning stock price and the stock price after the event. This data confirmation is labor-intensive, while the calculations proved rather straightforward.

This research has the following research question: do SM based financial disclosures improve shareholder value in financial institutions? The Null Hypothesis $\left(\mathrm{H}_{0}\right)$ is: $\mathrm{SM}$ will not enhance shareholder value. $\mathrm{H}_{0}$ where $\mathrm{MS}$ is mean stock price of organizations using $\mathrm{SM}$ and $\mathrm{MN}$ is nonsocial media stock price mean:

$$
\mathrm{H}_{0}: \mathrm{MS}_{1}=\mathrm{MN}_{2}
$$

The Alternative Hypothesis $\left(\mathrm{H}_{\mathrm{a}}\right)$ is: $\mathrm{SM}$ will enhance shareholder value. $\mathrm{H}_{\mathrm{a}}$ where MS is mean stock price of organizations using SM and MN is nonsocial media stock price mean:

$$
\mathrm{H}_{\mathrm{a}}: \mathrm{MS}_{1}>\mathrm{MN}_{2}
$$

This research, being an event study, identifies the "trigger event" as the corporate Earnings Call. This study focused on publicly traded American financial institutions on either the New York Stock Exchange or the NASDAQ system. Since stock price was readily available and verifiable, researchers selected publicly traded companies for the study. Also, the relationship between spread and stock movement represented a real world cause and effect.

Financial institutions were selected due to their reliance on technology. Financial institutions utilize large operating systems for monitoring customer accounts, transactions, security, marketing, and investor relations. Researchers wanted to see if these same institutions could benefit from an innovative use of a technology that is not required for operations.

The Securities and Exchange Commission has no standard format for release of financial statements. This means organizations can choose how they spread. Several different pieces of possible communication include: the earnings release, filing forms with the SEC, the public release of the $10-\mathrm{K}$ and $10-\mathrm{Q}$, and the release of the SM link (SEC, 2011). 
In order for an organization to be included in this study they needed to meet several predetermined criteria. These include:

1. They needed to be a financial institution

2. They needed to be publicly traded on either the NYSE or NASDAQ system

3. They needed to disseminate financial results the same day as the earning release

4. They needed to use either the Facebook or Twitter SM platforms

5. They needed to have at least 2,000 followers

The researchers could identify seventeen such organizations that met these criteria. The sample size for this study is thirty-four while the firm-quarter observations equal one hundred thirty-six. This includes seventeen organizations that use a SM platform to disseminate information and seventeen similar sized organizations that do not use SM. These organizations were monitored over an entire year. By using four quarterly releases and a matching set of financial institutions that do not use SM, the sample size grew significantly. This sample exceeds the thirty needed in a sample size for a normal distribution assumption. The margin of error was established to be $5 \%$ or 0.05 based on the research standard.

The calculation for sample size is: $17 \times 2 \times(1+3)=136$

Several items of data were needed to complete this analysis. These include daily stock prices and the Beta ratio for those organizations. Stock prices came from Measuringworth.com. Beta information came from Yahoo!Finance.com. The formula to decide stock price increase is as follows:

$$
R_{d}=\frac{P_{c}-P_{o}}{P_{o}}
$$

Notation:

$\mathrm{R}_{\mathrm{d}}=$ Daily Return

$\mathrm{P}_{\mathrm{c}}=$ Price of a share at daily closing

$\mathrm{P}_{\mathrm{o}}=$ Price of a share at daily opening 
This formula calculates Daily Return $(\mathrm{Rd})$. The numerator measures stock price at close of the day, minus the stock price at opening of the day. The stock price at the opening of a day comprises the denominator. $\mathrm{Rd}$ is the percent increase between the day's beginning stock price and the day's ending stock price.

Daily Return alone is not enough evidence of event impact. For example, what happens in the event that overall stock market happened to either increase or decrease on the day of a trigger event. To compensate, Abnormal Returns should be used. Abnormal Returns are the returns in excess of the normal Rd. Abnormal Returns are calculated by comparing the $\mathrm{Rd}$ to a standard normal return. One commonly used measurement is the organization's Beta ratio (Beta, 2016).

Accounting researchers use an idea called CARs in settling relevance of an event. This event is called a "trigger event." CARs looks at the value of that "trigger event."

CARs comprises several items. First, CARs incorporates abnormal returns. For example, consider the situation of the stock market advancing ten percent on a given day and that same day a company's stock also advances ten percent. Researchers would imply the advance was based on systematic market advances rather than the trigger event. For this research, the normal return was identified as the organization's Beta (or normal market response). Alternatively, if the market advances ten percent while a company advances fifteen, it could be argued the advance is a result of the trigger event. Second, CARs looks at the cumulative nature of the change. For example, in this research the cumulative effect of the three days before the trigger event and the three days post trigger event.

The formula for CARs comparing organizations using SM and organizations not using SM, represents one for the three days preceding the SM release and the three days following the release, and is:

$$
\mathrm{CAR}_{\mathrm{S}}=\sum_{(0-2)}^{M N}\left(\left(\frac{P_{O-} P_{C}}{P_{O}}\right)-\beta_{\text {stock }}\right)-\sum_{(-3--1)}^{M S}\left(\left(\frac{P_{O-} P_{C}}{P_{O}}\right)-\beta_{\text {stock }}\right)
$$




\section{Notation:}

$\mathrm{CAR}_{\mathrm{S}}=$ Cumulative Abnormal Return for SM

$\beta_{\text {Stock }}=$ Beta of the individual stock

MS $=$ Medium of SM

$\mathrm{MN}=$ Medium of nonsocial media

$\mathrm{P}_{\mathrm{c}}=$ Price of a share at daily closing

$\mathrm{P}_{\mathrm{o}}=$ Price of a share at daily opening

This study used the open-to-close stock pricing. This means stock prices were recorded at both the beginning of day (roughly 9:30am) and the ending day (roughly 4:00pm). This removes overnight trading to ensure the price change is more directly related to the information spread. This research focused on the American economy as measured by the Dow Jones Industrial Average (DJIA) (Williamson, 2015). Stock prices for individual companies and the DJIA were obtained from the website measuringworth.com. This information included the previous day closing price and the current disclosure date end of day price (Morningstar, 2015) as needed by the methodology.

\section{FINDINGS}

This section provides the results of the research. These findings are completed by first reviewing the key items necessary in the data collection process. Next, the data was analyzed from a descriptive statistics standpoint using advanced analytics in the program Excel. Output was segregated based on the use of SM. The following table uses descriptive statistics comparing stock price changes for organizations using SM versus nonsocial media for the three days following the release: 


\begin{tabular}{lll} 
Quarter/Variable & Social Media & Nonsocial Media \\
& Mean Stock Price $(\%)$ & Mean Stock Price (\%) \\
\hline $4^{\text {th }}$ Q 2014 & -1.61 & 5.64 \\
$1^{\text {st }}$ Q 2015 & 0.70 & -17.79 \\
$2^{\text {nd }}$ Q 2015 & -1.20 & -0.59 \\
$3^{\text {rd }}$ Q 2015 & 29.96 & 30.64 \\
Total & 27.84 & 17.90
\end{tabular}

Table 1. SM stock price compared with nonsocial media for total percentage increase post "trigger event."

The data also shows that the stock price of organizations disseminating information using SM outperformed nonsocial media disseminators by a margin of almost ten percent. For the days following dispersion, the CARs stock value increased $27.84 \%$ for SM compared with the nonsocial media increase of $17.90 \%$.

During this reporting period, nonsocial media stock price outperformed the SM three of the four quarters. Those three quarters include: the fourth quarter of 2014 (5.64\% to $-1.64 \%)$, the second quarter of $2015(-0.59 \%$ to $-1.20 \%)$, and the third quarter of 2015 (30.64\% to $29.96 \%$ ). In the first quarter of 2015, SM stock price outperformed nonsocial media by a margin of $0.70 \%$ to $-17.79 \%$. The reason for this large variance is two institutions underperformed based on their history. The comparison of the organization to market expectations via abnormal returns was unfavorable.

The data was also analyzed using the least-squares regression. In this case, providing support to "Reject" the Null Hypothesis. This means there was a discernable difference between the two datasets of SM disseminators and nonsocial media disseminators. In other words, using SM to disseminate financial results did lead to a higher stock price. The difference can be seen using the tValue and the F-Value for the three-day period following dissemination. In fact, 
the overall Values were lower than the standard benchmark of 0.05 to reject the Null. When considering the t-Value, in SM disseminators were below in two of the four quarters. The F-Value, on the other hand, was less conclusive. This was a result of the F-Value being 0.048 and only being below the 0.05 threshold for two quarters. The following table uses descriptive statistics comparing stock price changes for organizations using SM verses nonsocial media for the entire six-day trading window following the release:

\begin{tabular}{lll}
\hline Quarter/Variable & Social Media & Nonsocial Media \\
& Mean Stock Price $(\%)$ & Mean Stock Price (\%) \\
\hline $4^{\text {th }} \mathrm{Q} 2014$ & -8.88 & 22.93 \\
$1^{\text {st }} \mathrm{Q} 2015$ & 13.43 & -11.51 \\
$2^{\text {nd }} \mathrm{Q} 2015$ & -0.07 & -2.53 \\
$3^{\text {rd }} \mathrm{Q} 2015$ & 50.81 & 44.99 \\
Total & 54.63 & 53.89 \\
\hline
\end{tabular}

Table 2. SM stock price compared with nonsocial media for total percentage increase during sixday trading window

The data shows organizations releasing results using SM outperformed those that did not. Using the six-day trading window, SM disseminators had average stock price returns of $54.63 \%$ while nonsocial media organizations returned $53.89 \%$. This represents a $0.74 \%$ difference. In the financial world, a small percentage change in stock price results in billions of dollars in market value.

Stock price outperformance occurred in three of the four quarters measured. Those three quarters include: the first, second, and third quarters of 2015. In only one quarter did nonsocial media entities outperform SM entities. This occurred in the fourth quarter of 2014. In this quarter, nonsocial media organization increased $22.93 \%$, while SM decreased $8.88 \%$. The reason for this large variance is two institutions became takeover targets, and one entity had significantly improved results. 
The data was also analyzed using the least-squares regression. In this case, provided support to "Fail to Reject" the Null Hypothesis. This means there was no discernable difference between the two datasets of SM disseminators and nonsocial media disseminators. This can be seen using the t-Value $(0.24)$ and the F-Value (0.17) for the six-day trading period. In fact, the overall Values were higher than the standard benchmark of 0.05 . When considering the t-Value, in SM disseminators were below in two of the four quarters. The F-Value, also concluded there was no difference between the data sets. For entities using SM, the following Table compares the three days before transmission to the three days after:

\begin{tabular}{lll} 
Quarter/Variable & Social Media (Prior) & Social \\
& Mean Stock Price $(\%)$ & Mean \\
\hline $4^{\text {th }}$ Q 2014 & -7.26 & -1.61 \\
$1^{\text {st }}$ Q 2015 & 12.73 & 0.70 \\
$2^{\text {nd }}$ Q 2015 & 0.47 & -1.20 \\
$3^{\text {rd }}$ Q 2015 & 20.85 & 29.96
\end{tabular}

Total 26.79

27.84

Table 3. SM stock price total percentage increase comparing prior and post "trigger event."

Comparing between the days before and after transmission supports the conclusion that using SM improves the CARs of an organization's stock price. In this analysis, stock value went up $27.84 \%$ the three days following distribution, while the result was not as strong the days before distribution at $26.79 \%$.

The data was also analyzed using the least-squares regression. In this case, provided support to "Fail to Reject" the Null Hypothesis. This means there was no discernable difference between the two datasets of SM disseminators and nonsocial media disseminators. This can be seen using the t-Value (0.09) and the F-Value (0.15) for the six-day trading period. In fact, the overall Values were higher than the standard benchmark of 0.05 . When considering the t-Value, in SM 
disseminators were below in two of the four quarters. The F-Value, also concluded there was no difference between the data sets.

\section{CONCLUSIONS AND FUTURE RESEARCH}

This research combines financial community and technology applications. It examined the impact between using SM to spread financial results and not using a SM platform. Reviewing the Null and Alternative Hypotheses, several conclusions result from this work, answering the question: do SM based financial disclosures improve shareholder value?

Overall, this research determined that it was useful for organizations to spread their financial statements using social networks. This is a result of, on all accounts, the CARs share value increasing because of SM in the three-day period following the dissemination. Four other conclusions were determined.

First, banks should employ a strategy for disseminating financial statements involving a SM platform. With little risk or investment, organizations could release their earnings to potential new investors. Once incorporated, the procedure becomes commonplace and reliable to the potential investor. The agency relationship enjoyed by management requires action in the best interest of shareholders. Organizations that adopt a distribution policy early will benefit from the compounded impact of multiple periods, even if those movements are small in the beginning.

Second, today organizations normally have a live earnings call. This earnings call is a live meeting where interested parties can either attend or videoconference in a meeting about entities' financial results. At the end of the earnings call, it is normal for an investor relations representative to press an electronic button that spreads the company's financial results to predetermined outlets. Often these outlets include Bloomberg Financial, Reuter's, and the company's web site. This recommendation involves adding several social network outlets, such as Twitter and Facebook, to those predetermined outlets.

Third, organizations that do not disseminate using social web should research the platform. Though sometimes new procedures seem risky, the likelihood of an organization suffering from adverse unintended results such as prosecution is 
unlikely. This is a result of the Securities and Exchange Commission's willingness to help organizations correct errors and ensure future compliance.

Fourth, this research should be reexamined as more organizations adopt social network policies consistent with this research. This follow-up targets the discovery of new and emerging trends. This research could be used to decide the future impact or adverse conditions.

This research has several benefits. First, this research increases understanding on whether adding additional digital channels of distribution of financial disclosures impacts the organization's stock price. Second, this research improves the body of knowledge on the topic of SM platform effectiveness for financial communications. This improvement looks into the capacity of the social web to reach potential shareholders.

There are several limitations to this study. One limitation includes the fact that very few financial institutions use SM for financial dissemination. Though this is proof for the need of the research, the size forced a smaller than desired sample size. Another limitation is the fact that stock price is not determined on one bit of information. On a daily basis several factors may contribute to changes in stock price. This research had to assume no other internal corporate information impacted the share price. A further limitation is stock price change may not necessarily be measurable in a three-day period. Not all investors react in quick manner. This research was unable to follow investors and monitor the decision making process.

This study has many opportunities for future research. One such topic could focus research on specific social networks, including Twitter or Facebook to discover if one platform is more effective than others. Expanding this further can be carried out through advanced academic analytics. Also important is studying the power of technology adoption to find out if the change in stock price ties to an organization's innovativeness or the broadcast platform. Qualitative research to better understand what investors want in financial disclosures can also be completed. 


\section{REFERENCES}

ALEXANDER, R.; Gentry, J. (2014): Using social media to report financial results, Business Horizons, Vol. 57, No. 2: 161-167. https://doi.org/10.1016 /j.bushor.2013.10.009

BARNES, N. G.; LESCAULT, A. M.; WRIGHT, S. (2013): Fortune 500 are bullish on social media: Big companies get excited about Google+, Instagram, Foursquare and Pinterest. Available at: www.umassd.edu/cmr/ socialmedia research/2013fortune500/

BETA. (2016): In Financial-dictionary.com. http://financial-dictionary.thefree dictionary.com/Beta.

BLANKESPOOR, E.; MILLER, G. S.; WHITE, H. D. (2014): The role of dissemination in market liquidity: Evidence from firms' use of Twitter, The Accounting Review, Vol. 89, No. 1: 79-112. https://doi.org/10.2308/accr-50576

BOYLAN, D. H. (2016): The innovative use of Twitter technology by bank leadership to enhance shareholder value, ProQuest.

CHARLIER, M. (2013): Social media: Cause for pause, IR update, 9, 7-10.

DEISER, R.; NEWTON, S. (2013): Six social-media skills every leader needs, The McKinsey Quarterly, Vol. 1, 62-75.

HEGGESTUEN, J.; DANOVA, T. (2013): Brand presence: How to choose where to be on social media, without breaking your budget, Business Insider.

KAPLAN, A. M.; HAENLEIN, M. (2010): Users of the world, unite! The challenges and opportunities of social media, Business Horizons, Vol. 53, No. 1: 59-68. https://doi.org/10.1016/j.bushor.2009.09.003

LI, X.; XIE, H.; CHEN, L.; WANG, J.; DENG, X. (2014): News impact on stock price return via sentiment analysis, Knowledge-Based Systems, Vol. 69: 14-23. https://doi.org/10.1016/j.knosys.2014.04.022

MORGAN, J.D. (2013): SEC permits social media use for corporate disclosure. National Investor Relations Institute. http://www.niri.org/Main-Menu-Category/ resource/publications/Executive-Alert/2013-Executive-Alert-Archive/SECpermits-Social-Media-Use-for- Corporate-Disclosure-.aspx. 
MORNINGSTAR. (2015): Ticker price. http://www.msn.com/en-us/money/stock details/fi-126.1.IBKC.NAS?ocid=INSFIST10.

RAMASSA, P; DI FABIO, C. (2016): Social media for investor relations: A literature review and future directions, The International Journal of Digital Accounting Research, Vol. 16: 117-135.

REILlY, A. H.; HYNAN, K. A. (2014): Corporate communication, sustainability, and social media: It's not easy (really) being green, Business Horizons, Vol. 57, No. 6: 747-758. https://doi.org/10.1016/j.bushor.2014.07.008

SHUMAKER, R. P.; CHEN, H. (2009): Textual analysis of stock market prediction using breaking financial news: The AZFinText system, ACM Transactions on Information Systems, Vol. 27, No. 2: 1-19. https://doi.org/10.1145/1462198.1462204

SEC Clarifies Social Media Use and Reg FD Compliance. (2013): http://www.goodwinprocter.com/Publications/Newsletters/Client-Alert/2013/0.

SEC How to read a 10-k. (2011): http://www.sec.gov/answers/reada10k.htm.

TETLOCK, P. C. (2007): Giving content to investor sentiment: The role of media in the stock market, The Journal of Finance, Vol. 62, No. 3: 1139-1168. https://doi.org/10.1111/j.1540-6261.2007.01232.x

THORING, A. (2011): Corporate tweeting: Analysing the use of Twitter as a marketing tool by UK trade publishers, Publishing Research Quarterly, Vol. 27, No. 2: 141-158. https://doi.org/10.1007/s12109-011-9214-7

THORNTON, N. (2015): Is market volatility spooking 401(k) investors? Benefits Selling, Breaking News, 3.

WILLIAMSON, S. (2015): Daily Closing Value of the Dow Jones Average, 1885 to Present, MeasuringWorth.com, http://www.measuringworth.com/DJA/ 